\title{
Genre et variations stylistiques en sémantique textuelle
}

\author{
Christophe Gérard \\ LPL / Université de Provence \\ christophe.gerard@univ-provence.fr
}

\begin{abstract}
Malgré leur importance pour l'étude des arts du langage et la linguistique du texte en général, les rapports entre genre et style individuel demeurent peu étudiés, même dans des domaines aussi propices que la poétique et la stylistique.

Les méthodes de la linguistique de corpus assurent à présent la mise en oeuvre empirique de cette problématique aux paliers du mot et de la phrase. Plus précisément, comme elle reflète la diversité des usages oraux et écrits d'une langue historique, l'étude des variations lexicales et morphosyntaxiques dues aux discours et aux genres ${ }^{1}$ permet d'apprécier, par contraste, les régularités qui relèvent en propre de l'individuel. La notion de style s'identifie alors à celle d'idiolecte, pour un empan qui va des unités lexicales aux phénomènes transphrastiques. Au-delà, au palier du texte, les rapports entre genre et style intéressent les conditions posées par les normes traditionnelles du discours sur la singularisation de l'expression individuelle. Qu'en est-il en sémantique des textes?
\end{abstract}

Cette étude envisage le cas particulier du poème en prose et analyse, sur la base de propositions méthodologiques, les tendances de composition sémantique qui caractérisent la pratique de ce genre chez Gérard Macé (1946).

\section{Style, sens et textualité}

\subsection{Analogies définitionnelles et tâches descriptives}

Genette souligne très justement que toute identification et qualification d'un style (d'époque, d'école ou individuel) «déterminent un modèle de compétence capable d'engendrer un nombre indéfini de pages conformes à ce modèle » (1991 : 136). Or cette conception du style paraît tout autant s'appliquer au genre, dans la mesure où on y voit un « programme de prescriptions positives et négatives (et de licences) qui règlent la production et l'interprétation d'un texte $»^{2}$. À l'inverse, en revenant à la formulation de Genette, on serait tenté d'assigner cette dernière définition à la notion de style individuel. Non seulement, en effet, un tel style participe des modes de production du texte mais il en oriente aussi la réception en rendant prégnants des unités linguistiques et des phénomènes textuels qui autrement passeraient inaperçus. En ce sens, en deçà notamment de leur nature sociale/individuelle, genre et style ne se distinguent guère quant à leur statut de normes textuelles.

Pratiquement, la mise en évidence des régularités qui les différencient en corpus engage la description dans des tâches d'inégale indépendance. Alors que la caractérisation des genres nécessite leur comparaison au sein d'un même champ générique, celle des styles pose en particulier le genre comme un principe de différenciation, et présuppose ainsi la connaissance des normes génériques que met en jeu le corpus étudié (alors homogène de ce point de vue). Comme cette démarche subordonne le social à l'individuel, elle ne se confond pas avec une «stylistique des genres » dont le statut hybride conduit à ne pas privilégier les habitudes linguistiques de l'auteur ${ }^{3}$.

\subsection{Sémantique des genres et des styles}

Envisagée sous l'angle des composantes de la textualité ${ }^{4}$, la distinction entre style et genre apparaît essentiellement graduelle ( $v s$ catégorielle) : 
de la même façon que l'on peut définir un genre comme une interaction sociolectale entre composantes [du contenu et de l'expression], on peut définir un style comme une interaction idiolectale entre composantes. Cette interaction est d'un rang inférieur par rapport au genre, car elle intéresse des corpus moins étendus, mais en revanche ses prescriptions sont plus systématiques et plus fortes (Rastier $2001: 180$ )

Appuyée à ce dispositif théorique, la différenciation d'un style par les normes d'un genre se complique néanmoins de divers cas de figure, qu'on doit à l'existence de fonctionnements génériques multiples, dont certains mettent à l'épreuve l'analyse en composantes. C'est notamment le cas du poème en prose. D'ordinaire définit selon des critères très génériques comme un "texte poétique court, autonome et autotélique $»^{5}$, il apparaît dénué de prescriptions d'ordre thématique, narratif ou énonciatif, et ne définit donc pas d'interaction sociolectale au plan du contenu. Qu'une telle indétermination soit possible donne à imaginer, à titre heuristique, différentes façons pour un style de s'affirmer sous le régime textuel des composantes sémantiques. Dans cette perspective, les rapports entre genre et style rencontrent quatre cas de figure :

\section{Interaction sociolectale définie}

1a - On n'observe pas de régularités qui accuseraient une spécification idiolectale de l'interaction en question au sein du corpus d'étude (oeuvre littéraire, philosophique, etc.). Le style ne module pas son identité sur les prescriptions sémantiques du genre d'accueil et par conséquent la description des particularités individuelles devrait logiquement se poursuivre dans d'autres directions (cf. infra 3).

$1 \mathrm{~b}$ - On observe des régularités thématiques, narratives ou énonciatives justiciables d'un modèle individuel de production des textes. La description rend alors compte d'un style en tant qu'il se différencie sur un fond de caractéristiques génériques.

\section{Interaction sociolectale indéterminée}

$2 \mathrm{a}$ - On n'observe pas d'interaction régulière entre composantes. À nouveau, il faudrait enquêter selon d'autres perspectives pour dégager les particularités individuelles recherchées.

$2 \mathrm{~b}$ - Le corpus présente en tout ou partie des régularités idiolectales significatives. Elles indiquent une forme d'appropriation individuelle où, pour une oeuvre donnée, la sémantique du genre le cède à la sémantique d'un style.

Les cas $2 \mathrm{a}$ et $2 \mathrm{~b}$ correspondent à l'indétermination sémantique que nous avons illustrée avec le poème en prose. À l'inverse, les cas 1a et $1 \mathrm{~b}$ présupposent des prescriptions au plan du contenu, vis-à-vis desquelles s'apprécient la singularité d'un mode de production et d'un mode d'anticipation du sens textuel (pour une réception familiarisée avec les textes ainsi mis en série). À cet égard, alors que $1 \mathrm{~b}$ localise la différence de degré entre style et genre que signalent les propositions de Rastier, $2 \mathrm{~b}$ réaliserait lui un investissement stylistique « catégoriel ». Les analyses suivantes illustrent ce dernier cas de figure sur un corpus poétique réduit. 


\section{Poème en prose et variations stylistiques}

\subsection{Problématique}

L'oeuvre de Gérard Macé est réputée se soustraire aux catégories génériques classiques. Cette particularité concerne non seulement sa poésie mais aussi d'autres usages de l'écrit. Aussi la critique uset-elle d'habitude de guillemets lorsqu'elle considère Ex Libris (1980) comme un «essai » mêlant imagination personnelle et documentation érudite, ou encore Le dernier des Égyptiens (1988) comme une « biographie » qui explore la vie de François Champollion sur un mode thétique.

La spécificité de la poésie macéenne résulterait de même d'un "mélange de genres » (récit, poésie, essai) $^{6}$; d'un « 'brouillage' des genres » (entre prose et poésie) ${ }^{7}$ ou encore de l'invention d'une « forme » propre (Asso 2001 : 28). À la différence de cette communauté de vues, une minorité suggère pourtant d'assimiler cette poésie au genre du poème en $\operatorname{prose}^{8}$. La critique se présente ainsi comme divisée entre le refus et l'assomption d'un "genre » unique. Toutefois ces divergences sur la catégorisation des textes ne sont peut-être qu'apparentes.

Le poème en prose témoigne en effet dès son origine (1750-1850) des symptômes rencontrés par la critique macéenne : (i) comme le signale l'équivoque notoire de la locution poème en prose, il est un lieu privilégié de la contestation des frontières entre poésie et prose ; (ii) Nathalie Vincent-Munnia note par ailleurs que, dans la première moitié du XIX siècle, «Le poème en prose apparaît [...] comme un genre indéfinissable, ne pouvant être soumis à aucune caractérisation générale et définitive » (Vincent-Munnia 2003 : 562) ; (iii) en outre, Sandras a soin de relever des usages variés du genre qui « contraint d'admettre plusieurs modèles du poème en prose ", à savoir un modèle narratif, un modèle descriptif, un modèle musical ou euphonique, un modèle expérimental, enfin un modèle " proche du carnet ou de l'essai ${ }^{9}$; (iv) enfin, à l'origine "Les auteurs eux mêmes ne semblent pas pouvoir trouver une dénomination générique adéquate à leurs textes [...]. La même absence de classification générique nette marque le discours de la critique et de la réception contemporaine de ces textes.» ${ }^{10}$

Sur ce fond éclairci du débat, nous opposerons aux thèses du mélange et de l'exclusion des "genres » celle d'un genre unique attesté, en montrant que Macé n'a pas trouvé une «forme » déliée de la notion de genre, mais différentes manières de pratiquer le poème en prose. On cherchera par là à rendre compte des tendances de composition qui exploitent l'indétermination sémantique de ce genre.

\subsection{Analyses}

Restreint à 25 textes, le corpus d'étude couvre une période postérieure aux trois premiers recueils de Macé, Le jardin des langues (1974), Les balcons de Babel (1977) et Bois dormant (1983), et ne retient pas les premières parties de La mémoire aime chasser dans le noir (1993) qui relèvent pour l'essentiel de l'essai bref et de l'aphorisme. Il se compose ainsi de la troisième partie de ce dernier recueil, du recueil complet Le singe et le miroir (1998) ainsi que de trois poèmes d'abord publiés séparément, Pierrot, valet de la mort (1986), Tête-bêche (1987) et La forêt qui se met à marcher (1991) ${ }^{12}$.

Analogues au plan de l'expression, ces poèmes marquées par l'hypotaxe se particularisent tous par une présentation en alinéas qui segmente le texte en autant de périodes (pour un exemplaire cf. infra 2). Ces aspects marquent l'unité de facture des poèmes étudiés. Au plan du contenu, la métaphore continuée trame souvent la constitution du sens. Surtout, la plupart se laissent ramener à trois types d'interaction entre composantes. Nous choisissons de les nommer par commodité vie, songe et médiation pour indiquer la spécificité thématique, narrative et/ou énonciative de différentes «stylisations individuelles » ou appropriations sémantiques du poème en prose. 


\subsubsection{Caractères sémantiques des méditations}

Onze textes exemplifient des «méditations »: La chambre interdite, Suite royale, Hôpital de jour, La peur des miroirs, Au dieu du voyage, Hôtel de l'univers, Orphée qui se retourne, Le vent est à la prose, Le singe et le miroir, L'espoir est une étoile filante..., Le royaume des morts.

- Aspects narratifs et thématiques. Leur mode de production rappelle la technique médiévale de l'integumentum ou reprise et intégration rhétorique de mythes. Une large part des poèmes tire en effet sa matière narrative de figures mythiques ou de types littéraires. Ainsi la figure connue de Gaspard Hauser se superpose au mythe de Narcisse dans La peur des miroirs; le personnage décrit dans Au dieu du voyage représente Hermès ; Orphée revient sans Eurydice dans Orphée qui se retourne; Ulysse et les Sirènes voisinent avec tout un bestiaire merveilleux dans Le vent est à la prose; le début de Le singe et le miroir transfigure le mythe dogon des Jumeaux puis celui de l'Enfant sauvage né au siècle des Idéologues, etc. Ces transpositions de l'imaginaire collectif dans l'univers macéen servent de support à l'expression poétique d'une «thèse » plus souvent illustrée qu'explicitée (infra).

D'une façon constante, chacune des méditations présente un point de vue réflexif portant sur un « Nousmêmes ». Cette instance de la communication représentée est lexicalisée par un Nous généralement inclusif que les textes suggèrent d'identifier à la société occidentale moderne (entre la fin du XIX ${ }^{\mathrm{e}}$ et l'extrême fin du $\mathrm{XX}^{\mathrm{e}}$ siècle). $\mathrm{Y}$ invitent des termes, expressions, citations et allusions fonctionnant pour le lecteur à la manière de chronotopes tels que «cravate», "Kantor», «journal», « néon », «nos lumières celles des vitrines", "des oreilles absolument modernes et sourdes absolument», etc. Les déictiques personnels et temporels des méditations sont généralement interprétés ainsi, même s'il arrive aussi que le propos thétique, en même temps qu'il contribue à l'assimilation de ce Nous à l'Occident moderne, précise l'identité du foyer énonciatif. Par exemple dans Le vent est à la prose le thème de la poésie a pour corrélat un Nous, exclusif, qui renvoie aux acteurs de la modernité poétique.

- Des poèmes thétiques. Le royaume des morts et surtout La chambre interdite renvoient respectivement à d'autres textes de Macé qualifiables d'essai (bref et long) et d'aphorisme : le sixième paragraphe de Le royaume des morts évoque Le goût de l'homme ${ }^{13}$; La chambre interdite est un centon tout entier composé de passages de la première partie de La mémoire aime chasser dans le noir. Cette intertextualité du poème en prose avec les genres pragmatiques cités est symptomatique du caractère thétique des méditations, au sens où elles impliquent la prise en charge énonciative d'un propos objet d'un jugement évaluatif.

Le propos peut être explicite comme dans Suite royale («Les rois ne sont plus rois de droit divin, ils sont les servants de nos désirs »), ou indiqué comme dans Le dieu du voyage ("nous ne voyagerons plus grâce à Virgile dans l'enfer d'une autre vie ») ou dans La chambre interdite, un poème qui reprend le topos du réalisme mensonger de la photographie. Mais le plus souvent l'interprétation n'accède pas immédiatement au propos : La peur des miroirs et Hôpital de jour disent la laideur de l'écriture et du monde via la Beauté personnifiée ${ }^{14}$; Le vent est à la prose vise le modernisme en poésie; Le singe et le miroir regrette la parole perdue des origines, etc.

La modalisation évaluative est ici constante. De fait, si La peur des miroirs donne à re-parcourir les traits caractéristiques de Gaspard Hauser, c'est autant pour exploiter leur valeur symbolique qu'avec l'intention d'établir la comparaison finale, dépréciative : «c'est nous-mêmes» (164). Plus exactement, les jugements portés reposent sur des procédés métaphoriques bien plus que sur une argumentation articulée (en particulier par car et mais). Enfin, assumées par un Je implicite, les évaluations négatives sont dirigées vers l'acteur collectif Nous-mêmes et une situation historique — la Nôtre.

- Poèmes en prose " essayistes». L'ensemble de ces particularités signale la fonction polémique de ce foyer énonciatif. $\mathrm{Du}$ point de vue des actes de langage, un tel dispositif textuel peut susciter 
l'identification du lecteur réel au foyer interprétatif (également implicite au sein de la communication représentée). Plus précisément, par leur modalisation négative d'un propos défini, ces textes semblent dessiner en creux la place d'un énonciataire critique qui, en d'autres situations de discours, pourrait aller jusqu'à engager la controverse (parodie, lettre ouverte, etc.). Malgré tout une telle ouverture pragmatique demeure ici virtuelle, à la différence de l'essai, par exemple, dont le régime communicationnel appelle la réalisation d'une réception active (maintenue privée ou rendue publique). Le type de la méditation opère néanmoins en deçà et leur dimension thétique, source de leur coloration « essayiste », oblige au fond à retenir une conception affaiblie de l'autotélisme invoqué comme critère de définition du poème en prose.

\subsubsection{Caractères sémantiques du songe}

Relèvent du songe macéen Un ange passe..., La mémoire aime chasser dans le noir, Sommeil levant je

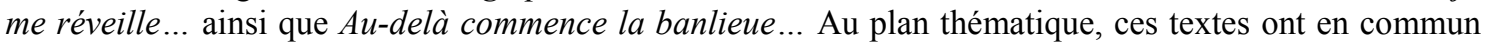
d'actualiser le champ lexical de la parenté et d'être dominés par une tonalité funèbre. Leur disposition linéaire est dominée par l'énumération. On se concentrera ici sur d'autres aspects de la textualité.

- Perception et souvenir. Du Bellay et Ronsard ont illustré le Songe d'une façon qui renvoie directement au Canzone dei visioni ${ }^{15}$. Au début du songe VIII de Du Bellay

$$
\begin{aligned}
& \text { Je vis un fier Torrent, dont les flots escumeux } \\
& \text { Rongeoient les fondements d'une vieille ruine : } \\
& \text { Je le vy tout couvert d'une obscure bruine, } \\
& \text { Qui s'eslevoit par l'air en tourbillons fumeux }{ }^{16}
\end{aligned}
$$

la construction Je + VOIR explicite un trait identificatoire de la vision, autre nom du songe ${ }^{17}$. Chez Macé celui-ci manifeste de même une corrélation privilégiée entre la modalité de la perception et le foyer énonciatif représenté par Je. Ce procédé est utilisé sous cette forme dans Un ange passe... mais aussi selon la modalité auditive («j'entends un bruit de vaisselle qui brise le rêve ») :

Un ange passe, et pendant que les conversations se taisent autour de la table, il revient accompagné de son jumeau, les ailes collées par la poussière et la sueur. Mais personne ne saurait dire aujourd'hui lequel des deux a levé lentement sa main baguée pour nous adresser un signe plein de grâce et de vulgarité à la fois, un signe annonciateur et louche.

Je revois le visage des jumeaux dans l'embrasure de la porte (celui qui tire la nappe en effaçant nos souvenirs, et celui qui transmet la tradition comme un mot de passe), chaque fois qu'en cherchant le sommeil j'arrange des mariages où sont invités les morts : des garçons d'honneur aux souliers vernis, les vieillards en chemises sans col, l'aïeule coiffée de noir depuis qu'elle a fauté.

Je revois «l'oncle matinal», les cousins près de leur sous et ceux qui n'ont pas pu avoir d'enfants, je revois Roger d'Orléans et Melaine de la Courberie anoblis par l'usage familial, et par le temps qui leur a fait l'aumône d'une particule. Puis vers la fin du banquet, à l'instant où les fiançailles vont devenir des noces d'or, et l'aïeule une infante, j'entends un bruit de vaisselle qui brise le rêve : à la place des deux anges qui n'ont laissé aucun message, et qui n'apparaîtront plus 


\begin{abstract}
ensemble sur la terre, je ne vois que des plumes comme après un combat de coqs, et la volaille qu'on engraisse pour le prochain mariage.

Enfin le photographe avec ses airs de faux prophète, et sa lanterne allumée en plein midi pour nous annoncer la nouvelle de notre mort.
\end{abstract}

Surtout, la lexicalisation « revois » se rapporte à un événement passé. C'est l'indice d'une différence majeure par rapport au songe traditionnel. C'est en effet la triple corrélation de la fonction du Souvenir ( $v s$ Imagination), de la modalité perceptive et du Je qui identifie le songe macéen. Chez l'auteur de $L a$ mémoire aime chasser dans le noir le songe est ainsi davantage acte de remémoration qu'acte d'imagination productive ; cette présence de l'activité mnésique faisant directement écho au thème de la Mémoire, central dans cette oeuvre.

- Mode du récit et impression référentielle. Le songe se caractérise par une impression référentielle de type onirique, qui peut être produite au moyen d'antilogies. Par exemple, dans «à l'instant où les fiançailles vont devenir des noces d'or, et l'aïeule une infante », d'une part le contexte transformatif («devenir ») accueille paradoxalement un état /antérieur/ (« infante ») en position résultative. D'autre part, «à l'instant où » vient rendre saillant le trait /imminence/ dans «les fiançailles vont devenir des noces d'or», impliquant ainsi une transformation ponctuelle alors même que la relation qui lie « fiançailles » à « noces d'or » est durative.

Relevant de cette rationalité onirique (i.e. ni causale ni finalisée), le récit fragmentaire qu'implique le songe macéen n'appelle pas à restituer les moments manquants d'une intrigue énigmatique. Par exemple, dans Un ange passe... le retour de l'Ange est marqué par une transformation (ses ailes sont « collées par la poussière et la sueur ») qui demeure sans origine. Certes l'intelligence narrative peut tenter d'expliquer certaines ellipses supposées. Aussi n'apparaît-il pas déraisonnable d'imaginer ici une lutte en lien avec les fonctions antithétiques de ces Anges de la mémoire ( « celui qui tire la nappe en effaçant nos souvenirs, et celui qui transmet la tradition comme un mot de passe »). Mais généralement la succession des d'actions ( « revient », « puis vers la fin ») s'émancipe du récit canonique (clôture, symétrie et causalité) et aucune organisation narrative globale ne peut être constituée.

\title{
2.2.3 Caractères sémantiques des Vies
}

Présente dans l'œuvre de Macé, en particulier dans les « essai-fictions » des Vies antérieures, on retrouve la vie dans les poèmes Parade nuptiale, Femmes sans tête, Tête-bêche, La forêt qui se met à marcher et Entre le théâtre et les bois... Certains textes à la première personne (Opéré de quelque grosseur...; De la partie fermée $d u$ théâtre...; La leçon d'anatomie), de facture autobiographique, se rapprochent naturellement de ces récits de vie, mais sans en relever strictement.

— Les vies et les songes. Ils contrastent à divers égards. Les premières appliquent la modalité ontique du /réel/ aux acteurs du récit, alors que le mode mimétique des seconds se traduit par une saturation du texte par l'irréel. De plus, on n'y observe pas d'interactions entre le narrateur et les acteurs, à la différence des songes (notion de narrateur-personnage). Enfin, à la narration homodiégétique du songe s'oppose la narration hétérodiégétique de la vie centrée sur des événements passés et actuels d'au moins un acteur humain.

- L'espace profond du souvenir. Cet acteur, qui appartient à la classe des Défunts, est toujours identifié (les Parents dans Parade Nuptiale, le Nourrisson dans Femmes sans tête) et parfois désigné par un nom propre (ex. Arthur Meslin et Gervaise Vidor dans La forêt qui se met à marcher, Saturnin dans Tête- 
bêche). La structure récurrente qui le caractérise corrèle un IL à une modalité spécifiée par ce que nous avons appelé la fonction du Souvenir ; comme si la mémoire propre des personnages était donnée responsable du récit (par ex. dans Femmes sans tête «C'est dans une quinte de toux qu'il retrouve une impression d'enfance »). Il est clair toutefois que l'accès à l'histoire individuelle des personnages se fait par la médiation du foyer énonciatif (Je). De sorte que lui-même connaît une actualisation de la fonction du Souvenir, qui le cède parfois à l'Imagination ${ }^{18}$. Une spécificité remarquable des vies réside dans cette inclusion des fonctions du Souvenir : Je se souvient d'un Il qui se souvient de son passé.

— Thématique vitale et tonalité funèbre. Ce qui unit par ailleurs tous ces poèmes est une thématique du cycle vital ${ }^{19}$. On note au hasard les chronotopes « dans le ventre de sa mère », « nourrisson », « enfance », « en tremblant comme une feuille » (vieillesse), « son fantôme » (résurrection). Mais le parcours du cycle n'a rien de linéaire et de duratif. Par ailleurs, la narration de ces vies anecdotiques porte sur des gens de petite condition (ex. le bleu de travail et le tablier) à l'image de la Mère de Parade nuptiale, cette "héroïne d'une vie brève qui s'écroula un soir sur le seuil de sa maison». Ce passage marqué par l'aspect /perfectif/ («brève », «s'écroula », « soir », « seuil ») exemplifie enfin le ton de ces vies hantées par le deuil : en qualifiant globalement le thème de la vie, l'aspect/perfectif/ contribue ainsi à produire la coloration funèbre qui domine les poèmes.

\section{Synthèse et perspectives}

Sur la base d'équivalences sémantiques globales, nous avons tenté de rapprocher des textes identifiés par une facture typographique et syntaxique analogue et appartenant à un même genre. Leur description a permis de mettre en évidence des caractéristiques communes (thématiques, énonciatives et/ou narratives) qui, pour la majorité des vingt cinq textes examinés, constituent des sortes de sous-ensembles et légitiment de fait des formes textuelles stables propres à Gérard Macé. Ainsi, alors que le songe s'oppose « en interne » à la vie comme à la méditation, il paraît même se singulariser par rapport à sa tradition poétique (Du Bellay, Ronsard).

Ces formes, qui n'ont le statut de type textuel qu'au sein de cette poésie, se définissent par des interactions spécifiques entre composantes. Ce sont elles qui, dans le cadre d'un genre dénué de prescriptions au plan du contenu, fondent l'existence de variations stylistiques. La méditation est de loin le type le plus exemplifié (onze poèmes), viennent ensuite la vie (cinq exemplaires) et le songe (quatre exemplaires). Les catégorisations hybrides de la critique macéenne se précisent alors : de même que la dimension thétique des médiations rapproche ces poèmes de l'essai, de même la dimension narrative du songe et de la vie conduit naturellement à employer l'étiquette «récit». À vrai dire, ces formes témoignent ensemble d'une pratique plurielle (i.e. ni uniforme ni disparate) du poème en prose, cette pluralité de manières déclinant un style de composition qui se positionne entre la contingence de la création poétique et l'emploi de procédés systématiques.

Exploitant les catégories rhétoriques du portrait ou du tableau, cinq textes sont toutefois rétifs aux regroupements effectués. À l'exception de Pierrot valet de la mort, ils appartiennent au dernier recueil de Macé, Le singe et le miroir. Certes Le roman des jumeaux et Le porteur de lanterne, où un Je confie son passé et médite sur l'origine, tiennent un peu de la vie et de la méditation. De même Assis sous un auvent..., Pierrot valet de la mort et $A$ l'intérieur du navire... mettent en scène un acteur unique (l'Emigrant, le Souffleur, le Capitaine, respectivement) qu'un sort dramatique rapproche des vies. Mais ces poèmes ne sont pas en toute rigueur assimilables aux formes textuelles dégagées. Ce genre de difficulté appelle à prolonger l'étude et la recherche en complétant la différenciation du style par le genre au moyen d'autres formes de caractérisation sémantique des particularités individuelles.

Nous avons fait ailleurs des propositions en ce sens qui s'appliquent aux façons régulières de constituer le sens au sein d'un corpus attribué à un énonciateur unique. En effet, une oeuvre peut se singulariser à différents paliers et donc se caractériser sous différents angles : 1/ le palier du texte situe les interactions idiolectales entre composantes ; 2 / celui de la période intéresse des structurations remarquables entre 
signifiés, envisagés dans leur linéarité (rythmes sémantiques, etc.) ; 3/ au palier du mot l'oeuvre constitue les significations propres à l'auteur ; 4/ enfin, à ces différents paliers, la constitution du sens peut épouser un même schème. Ainsi chez Macé un contraste fort unifie les paliers du mot (ex. cercueil de verre), de la période (ex. un rat qui voulait devenir une étoile) et du texte (figure macéenne des Jumeaux antagonistes ; opposition thématique entre culture savante et culture populaire, etc.).

Au-delà, l'analyse sémantique sert une caractérisation textuelle qui doit rendre compte de l'interaction entre le plan de l'expression et celui du contenu. Cela intéresse en particulier les trois recueils qui précèdent Bois dormant (Le jardin des langues, Les balcons de Babel et Bois dormant). Macé y voit une trilogie dont le mode d'écriture n'est pas sans évoquer les productions dites automatiques des Surréalistes. À la différence des recueils suivants, ils soumettent la lecture à un tempo soutenu par la suppression des points, des virgules et l'usage de l'énumération, notamment. Cette différence de facture soulève le problème des lignées stylistiques qui alternent dans la chronologie des parutions et dont la reconnaissance repose sur des indices de périodisation (typographiques, phoniques et morphosyntaxiques). Dans le cas présent, il s'agirait d'établir si la trilogie relève d'une lignée unifiée (pour une suite de textes, correspondance d'une même facture et d'un même style sémantique) ou non (un style sémantique commun a des factures distinctes).

Répondre à cette alternative permettrait d'apprécier l'évolution d'un style en terme de continuité/discontinuité. Il reviendrait à une linguistique des styles unifiée aux deux plans du texte d'investir ce domaine de la variation stylistique.

\section{Bibliographie}

Asso F. 2001. «Lecture, rêve, mémoire », in S. Boucheron, J.-L . Lambel, N. Ragonneau (éd.), Images et signes. Lectures de Gérard Macé, Cognac, Le Temps qu'il fait : 23.

Bercot M., Seth., C., Collot M. 2000. Anthologie de la poésie française, Du XVIII au XXe siècle, Paris, Gallimard, Pléiade.

Bernard S. 1959. Le Poème en prose, de Baudelaire à nos jours, Paris, Nizet.

Boucheron S., Lambel J.-L., Ragonneau N. 2001. Images et signes. Lectures de Gérard Macé, Cognac, Le Temps qu'il fait.

Combe D. 1992, Les genres littéraires, Paris, Hachette.

— 2002. «La stylistique des genres », Langue française, 135, 33-49.

Conort B. 2001. Art. «Gérard Macé », in M. Jarrety (dir.), Dictionnaire de poésie, De Baudelaire à nos jours, Paris, PUF, pp. 451-452.

Genette G. 1991. Fiction et diction, Paris, Seuil.

— 2007a. «Sémantique et linéarité du texte. La place du rythme en sémantique des textes »in M. Ballabriga (dir.), Rythme et textualités, Éditions Universitaires du Sud.

—2007b. « Sémantique interprétative des styles », L'information grammaticale, 113, Paris-Louvain, Peeters.

- (à paraître). « Sens et régularités idiolectales en poésie » (Actes de la Journée d'étude Lorand Gaspar et la langue, 12 mai 2006, Université Rennes 2).

Macé G. 1991. Vies antérieures, Paris, Gallimard, coll. Le Chemin.

— 2002. Bois dormant et autres poèmes en prose, Paris, Gallimard, coll. Poésie.

Para J-B. 1987. «La poésie tombée dans la prose (entretien avec Gérard Macé) », L’Infini, 19, Paris, Gallimard : 7482.

Rastier F. 2001. Arts et sciences du texte, Paris, PUF.

Rastier F., Malrieu D. 2001. «Genres et variations morphosyntaxiques », Traitement Automatique des langues, vol. $42, n^{\circ} 2: 548-577$. 
Rastier F., Cavazza M., Abeillé A. 1994. Sémantique pour l'analyse, Paris-Milan-Barcelone, Masson.

Sabatier R. 1988. « Gérard Macé », Histoire de la poésie française, La Poésie du XXe siècle, Albin Michel.

Sandras M. 2001. «Poème en prose », in M. Jarrety (dir.), Dictionnaire de poésie, De Baudelaire à nos jours, Paris, PUF, pp. 611-613.

Vincent-Munnia N., Bernard-Griffiths S., Pickering R. (éds.). 2003. Aux origines du poème en prose français, Paris, Honoré Champion.

Wandelère F. 2001. «Les balcons de Babel », in Boucheron S., Lambel J.-L., Ragonneau N., Images et signes. Lectures de Gérard Macé, Cognac, Le Temps qu'il fait.

${ }^{1}$ Rastier, Malrieu 2001.

${ }^{2}$ Rastier et alii 1994 : 222. Ainsi définis les genres occupent une hiérarchie où l'on distingue, par ordre d'inclusion, «les discours (ex. juridique vs littéraire vs essayiste vs scientifique), les champs génériques (ex. théâtre, poésie, genres narratifs), les genres proprement dits (ex. comédie, roman " sérieux », roman policier, nouvelles, contes, mémoires et récits de voyage), les sous-genres (ex. roman par lettres)» (Rastier, Malrieu 2001 : 548).

${ }^{3}$ Qui « serait ainsi par excellence la discipline où convergeraient l'analyse (socio)linguistique et l'esthétique, assurant une médiation entre la stylistique de la langue de Bally et la stylistique des auteurs à la manière de Spitzer » (Combe $2002: 43)$.

4 En plus des composantes rythmique et prosodique, le plan de l'expression informe un espace médiatique (écrit/oral/polysémiotique). Au plan du contenu, quatre composantes sont susceptibles d'interagir : la thématique intéresse la construction des thèmes, topoï et motifs. La dialectique les actions et les acteurs du récit (canonique ou non). La dialogique recouvre pour l'essentiel la communication représentée (foyers énonciatif/interprétatif) et les évaluations modales (factuel/irréel, vrai/faux, euphorique/dysphorique, etc.). Enfin, la tactique rend compte de la position relative des unités sémantiques.

${ }^{5}$ Vincent-Munnia, Bernard-Griffiths, Pickering $2003: 445$. Ces critères connaissent un large consensus. Citons ce commentaire de Combe (1992) sur les «solides critères de définition » de Bernard (1959: 92-93) : « « Le poème en prose, je l'ai déjà dit, suppose une volonté consciente d'organisation du poème: il doit être un tout organique, autonome, ce qui permet de le distinguer de la prose poétique... ». Suzanne Bernard résume cette exigence par les critères d'unité, de gratuité et de brièveté. Par «gratuité », il faut entendre le fait que le poème en prose, à la différence de la prose poétique, n'est pas une «matière» à partir de laquelle «on peut construire aussi bien des essais, des romans, des poèmes ». En définitive, "plus que le poème en vers, le poème en prose doit éviter les digressions morales ou autres, les développements explicatifs - tout ce qui ramènerait aux autres genres de la prose, tout ce qui nuirait à son unité, à sa densité ».

${ }^{6}$ « Macé est l'auteur d'ouvrages inclassables qui tiennent à la fois de l'essai, du récit (volontiers autobiographique) et de la poésie : entre autres, Le Jardin des langues (1974), Bois dormant (1983), La mémoire aime chasser dans le noir (1993)» (Bercot, Seth, Collot $2000: 1243)$.

${ }^{7}$ Sabatier $1988: 748$.

${ }^{8}$ Conort considère que le recueil La mémoire aime chasser dans le noir relève « du poème en prose » $(2001: 452)$, et Wandelère note à propos des Balcons de Babel : «Les poèmes en prose qui sont au cœur du recueil sont comparables à ceux publiés en 1974 dans le Jardin des langues » (2001: 17).

${ }^{9}$ Sandras 2001 : 611-613. Il nous paraît toutefois bien exagéré de parler ici de «modèles du poème en prose » pour désigner en fait une mise en série de textes qui repose sur des types aussi transversaux que la description, la narration, etc.

${ }^{10}$ Ibidem. On notera que pour Macé non seulement la question des genres est «oiseuse » mais aussi que la connaissance du genre en général « est précisément ce qui empêche d'écrire » (Para 1987 : 7 ).

${ }^{11}$ Nous reviendrons en conclusion sur cette exclusion de méthode.

${ }^{12}$ Réunis en 2002 dans Bois dormant et autres poèmes en prose. Nos citations renvoient à cette édition. On indique les poèmes non titré par leurs premiers mots suivis de points de suspension. 


\begin{abstract}
${ }^{13}$ Le passage « Une autre réponse vient à l'esprit à propos des nomades : manger ses morts est le meilleur moyen de toujours les avoir avec soi, de ne jamais les abandonner, puisque l'estomac est une sépulture ambulante, qui dure autant que nous » (Macé G. 2002. Le goût de l'homme, Le Promeneur : 60) correspond à cet autre «Les derniers à sortir de la forêt [...] furent aussi les derniers à manger leurs morts [...]. En faisant de leur propre corps une sépulture, ces nomades se protégeaient eux-mêmes des âmes errantes, insatiables, toujours prêtes à réclamer leur dû aux survivants. » (Macé 2002 : 189-190).

14 «Dans l'hôpital qu'est devenu le vaste monde, derrière les baies vitrées où elle vient battre de l'aile, je reconnais quelque fois la beauté qu'on insulte » (p. 163). Ce passage évoque bien entendu le début d'Une saison en enfer.

15 «Le Songe, où l'onirique conduit à l'ironique relève de la même inspiration mais prend une tonalité plus directement prophétique. Le Songe n'est pas le récit d'un rêve véritable, mais une fiction imitée librement de la Canzone dei visioni de Pétrarque et nourrie de surcroît d'emprunts à l'Ecclésiaste et à l'Apocalypse » (Lestringuant F., Rieu J., Tarrete A.. 2000. Littérature française du XVIe siècle, Paris, PUF : 216-217).

${ }^{16}$ In Du Bellay 1974. Les regrets et autres ceuvres poétiques suivis des Antiquités de Rome. Plus un Songe ou Vision sur le mesme subject, Genève, Droz : 314.

${ }^{17}$ Citons aussi ces vers de Ronsard extraits des Folastries : ...Je voy deça, je voy dela, / Je voy mille bestes cornues, / Mille marmotz dedans les nues : / De l'une sort un grand Toreau, / Sur l'autre sautelle un chevreau : / L'une à les cornes d'un Satyre, / Et du ventre de l'autre, tire /Un crocodile mille tours (Raymond M. 1971. La poésie française et le maniérisme - 1546-1610, Genève/Paris, Droz/Minard : 179-180).

${ }^{18}$ Dans Femmes sans tête « je revois le petit écorché », dans Parade nuptiale « si je ne les ai pas vus je les imagine », ou encore à la fin de La forêt qui se met à marcher «Le cadavre d'Arthur est le premier que j'ai vu ».
\end{abstract}

${ }^{19}$ Soit : vie antérieure, naissance et mort, renaissance, résurrection et incarnation. 